\title{
Impact of Distribution Tariff Design on the Profitability of Aggregators of Distributed Energy Storage Systems
}

\author{
Niels Govaerts ${ }^{* \dagger \ddagger}$, Kenneth Bruninx ${ }^{* \dagger \ddagger}$ and Erik Delarue*† \\ *Department of Mechanical Engineering, KU Leuven, Leuven, Belgium \\ ${ }^{\dagger}$ EnergyVille, Genk, Belgium \\ $\ddagger$ VITO, Mol, Belgium
}

\begin{abstract}
The distribution tariff design, which is currently being overhauled in multiple European countries, is a significant part of a residential consumer's electricity bill. This paper studies how the tariff design influences the behavior of a strategic aggregator, of residential consumers with photovoltaics (PV) and energy storage systems (ESS), on a wholesale market. The aggregator-wholesale market interaction is formulated as a Stackelberg game. We show that the distribution tariff design impacts the strategic operation of the aggregator's PV and ESS and the accompanying cost savings the aggregator attains compared to a retailer of active consumers.
\end{abstract}

\section{INTRODUCTION}

Due to the decreasing costs of photovoltaics (PV) and energy storage systems (ESS), a significant share of residential consumers may install small-scale PV and/or ESS. Since residential consumers cannot typically participate directly in wholesale markets due to their limited capacity, aggregators may be needed to monetize the flexibility of these distributed ESS and PV systems through demand response. However, the electricity bill of residential consumers does not only contain the energy cost, but also other cost components, such as distribution costs. Since the distribution tariff is the largest component of the electricity bill of residential consumers, e.g. in Belgium [1], the distribution tariff design may significantly affect the viability of a residential aggregator.

Due to the increase in residential consumers with PV systems, European regulators are revisiting the distribution grid tariff design. Current volumetric tariffs cause welfare transfers between different consumer groups and threaten the ability of distribution system operators (DSOs) to recover their costs [2]. In the academic literature, multiple distribution tariff designs are proposed and analyzed. Jargstorf [3], e.g., analyzes capacity-based distribution tariffs. However, Schittekatte et al. [4] show that, for recovering sunk costs, the non-cooperative behavior of residential consumers under a capacity tariff can result in significant welfare losses and equity issues. Borenstein [5] argues that, from an efficiency and equity standpoint, a combination of fixed and volumetric charges is the least bad policy option for recovering sunk costs.

Strategic behavior of price-making agents, such as a plugin electric vehicle aggregator [6] or a virtual power plant consisting of consumers, ESS and wind turbines [7], in electricity markets is often formulated as a Stackelberg game, formulated as a bilevel optimization problem. The upper-level (UL) problem represents the problem of the strategic agent while the lower-level (LL) represents the anticipation of the UL agent of the market clearing. In these models, the strategic agents typically try to maximize their profit or minimize their costs.

In this paper, we bridge the gap between these strands in the literature by modeling the strategic participation of an aggregator, representing residential consumers with PV and ESS, in a wholesale market as a bilevel optimization problem, reflecting a Stackelberg game. The aggregator is the strategic agent in the UL who attempts to minimize the cost of procuring electricity for its consumers, taking the flexibility of consumers' ESS into account. The aggregator explicitly accounts for the impact of its actions on the distribution component in the consumers' electricity bill, which is typically not considered in aggregator-focussed work (e.g. [6], [7]) while distribution tariff design studies (e.g. [3]-[5]) typically do not consider aggregators. The split of the benefits of monetizing the consumers' flexibility between consumers and the aggregator is not taken into account in this paper.

In Section II, the methodology is detailed. In Section III, we discuss the impact of five distribution tariff designs on the benefit of the aggregator-consumer collaboration in a case study inspired by the Belgian power system. Finally, in Section $\mathrm{IV}$, a conclusion is formulated.

\section{Methodology}

The mathematical formulation of the bilevel model and its reformulation as a mixed integer linear program (MILP) is detailed in Sections II-1, II-2 and II-3. The presented models are based on the following assumptions: the aggregator only participates in the wholesale market, transmission and distribution constraints are disregarded and investment decisions are not considered. We study four types of consumers with an inelastic demand: (i) passive residential consumers supplied by a retailer (RP), (ii) active residential consumers supplied by a retailer (RA) who does not strategically operate their assets, (iii) active residential consumers supplied by a strategic aggregator (AG) and (iv) passive consumers who buy directly from the wholesale market (I) (e.g., industrial consumers). 
1) The aggregator's problem (UL): Assuming the aggregator and consumers collaborate, the objective of the aggregator is to minimize the costs of supplying electricity to its consumers, following Bruninx et al. [8]. Aside from the costs/revenues of buying/selling electricity on the wholesale market $\left(\lambda_{t} \cdot\left(w_{t}^{\mathrm{ag}}-i_{t}^{\mathrm{ag}}\right)\right.$ with $\lambda_{t}$ the wholesale electricity price), these costs also include the distribution costs distcosts ${ }^{\mathrm{ag}}$. The sum of energy and distribution costs is minimized in the UL objective (1) and will be referred to as operating costs of the aggregator in the remainder of this paper. Other cost components, e.g. transmission costs, are not considered.

$$
\begin{aligned}
& \text { Minimize } \quad \sum_{t \in \mathcal{T}} \lambda_{t} \cdot\left(w_{t}^{\mathrm{ag}}-i_{t}^{\mathrm{ag}}\right)+d i s t c o s t s^{\mathrm{ag}} \\
& \text { subject to } \\
& w_{t}^{\mathrm{ag}}-i_{t}^{\mathrm{ag}}=D_{t}^{\mathrm{ag}}+c h_{t}^{\mathrm{ag}}-d c_{t}^{\mathrm{ag}}-p v_{t}^{\mathrm{ag}}, \quad \forall t \in \mathcal{T} \\
& e_{t}^{\mathrm{ag}}=e_{t-1}^{\mathrm{ag}}+c h_{t}^{\mathrm{ag}} \cdot E C-d c_{t}^{\mathrm{ag}} / E D, \quad \forall t \in \mathcal{T} \backslash\{1\} \\
& e_{1}^{\mathrm{ag}}=E_{0}^{\mathrm{ag}}+c h_{1}^{\mathrm{ag}} \cdot E C-d c_{1}^{\mathrm{ag}} / E D, \\
& e_{T}^{\mathrm{ag}}=E_{0}^{\mathrm{ag}}, \\
& 0 \leqslant e_{t}^{\mathrm{ag}} \leq \overline{E^{\mathrm{ag}}}, \quad \forall t \in \mathcal{T} \\
& 0 \leqslant c h_{t}^{\mathrm{ag}} \leq C R \cdot \overline{E^{\mathrm{ag}}}, \quad \forall t \in \mathcal{T} \\
& 0 \leqslant d c_{t}^{\mathrm{ag}} \leq C R \cdot \overline{E^{\mathrm{ag}}}, \quad \forall t \in \mathcal{T} \\
& 0 \leqslant p v_{t}^{\mathrm{ag}} \leq L S_{t} \cdot C A P_{P V}^{\mathrm{ag}}, \quad \forall t \in \mathcal{T}
\end{aligned}
$$

Equation (2) defines the aggregated hourly withdrawal $w_{t}^{\mathrm{ag}}$ and injection $i_{t}^{\mathrm{ag}}$ of the aggregator which depend on the inelastic demand of its consumers $D_{t}^{\mathrm{ag}}$, the charging $c h_{t}^{\mathrm{ag}}$ and discharging power $d c_{t}^{\mathrm{ag}}$ of its aggregated ESS and the production of its aggregated PV systems $p v_{t}^{\mathrm{ag}}$. Constraints (3)-(5) determine the energy content of the ESS $e_{t}^{\mathrm{ag}}$ at each timestep considering cyclical boundary conditions (the energy content in the first and last timestep equals $E_{0}^{\mathrm{ag}}$ ). The parameters $E C$ and $E D$ denote the charging and discharging efficiency. Constraint (6) limits the energy content of the ESS to the installed capacity $\overline{E^{\mathrm{ag}}}$, while constraints (7)-(8) limit the charging/discharging power based on the C-rate of the ESS $(C R)$. Constraint (9) limits the PV production to the installed capacity $\left(C A P_{\mathrm{PV}}^{\mathrm{ag}}\right)$ multiplied by the load factor $\left(L S_{t}\right)$. The distribution costs depend on the distribution tariff design.

1) For a fixed tariff (FIX), the distribution costs are constant.

2) For a volumetric tariff with net-metering (VOL ${ }^{\text {net }}$ ), the distribution costs are proportional to the (positive) net withdrawal of a consumer, calculated over a whole year: distcosts ${ }^{\mathrm{ag}}=t a r^{\mathrm{net}} \cdot \max \left(\sum_{t \in \mathcal{T}}\left(w_{t}^{\mathrm{ag}}-i_{t}^{\mathrm{ag}}\right), 0\right)$.

3) Under a volumetric tariff on hourly offtake $\left(\mathbf{V O L}^{\mathbf{}}\right)$, consumers pay for their hourly withdrawal: distcosts $^{\mathrm{ag}}=\operatorname{tar}^{\mathrm{O}} \cdot \sum_{t \in \mathcal{T}} w_{t}^{\mathrm{ag}}$.

4) Under a volumetric tariff on hourly offtake and injection $\left(\mathbf{V O L}^{\mathrm{o} / \mathrm{i}}\right)$, consumers also pay when they inject: distcosts $^{\mathrm{ag}}=\operatorname{tar}^{\mathrm{o} / \mathrm{i}} \cdot \sum_{t \in \mathcal{T}}\left(w_{t}^{\mathrm{ag}}+i_{t}^{\mathrm{ag}}\right)$.

5) Under a capacity tariff (CAP), the distribution costs are proportional to the maximum hourly offtake or injection during a year: distcosts $s^{\mathrm{ag}}=\operatorname{tar}^{\mathrm{cap}} \cdot \max \left(w_{t}^{\mathrm{ag}}, i_{t}^{\mathrm{ag}}\right)$.
2) The market clearing problem (LL): The market operator aims to maximize the total surplus w.r.t. the bids and offers of the market participants. We assume that conventional generators offer their capacity at average generation cost $V C_{j}$, the aggregator bids its demand at the price cap $P^{\text {cap }}$ and its supply at a price of zero to ensure its bids are accepted and the retailers and non-residential consumers also bid their demand $D_{t}=D_{t}^{\mathrm{ra}}+D_{t}^{\mathrm{rp}}+D_{t}^{\mathrm{i}}$ at the price cap. The objective of the market operator then reads (dual variables of each constraint between brackets):

$$
\begin{aligned}
& \text { Maximize } \sum_{t \in \mathcal{T}} P^{\text {cap }} \cdot\left(w_{t}^{\mathrm{ag}}+D_{t}\right)-\sum_{j \in \mathcal{J}} \sum_{t \in \mathcal{T}} V C_{j} \cdot g_{t, j}^{\mathrm{t}} \\
& \text { subject to } \\
& w_{t}^{\mathrm{ag}}+D_{t}^{\mathrm{rp}}+D_{t}^{\mathrm{ra}}+D_{t}^{\mathrm{i}}+c h_{t}^{\mathrm{ra}}= \\
& \sum_{j=1}^{J} g_{t, j}^{\mathrm{t}}+g_{t}^{\mathrm{w}}+d c_{t}^{\mathrm{ra}}+p v_{t}^{\mathrm{ra}}+i_{t}^{\mathrm{ag}}, \quad \forall t \in \mathcal{T} \quad\left(\lambda_{t}\right) \\
& 0 \leqslant g_{t, j}^{\mathrm{t}} \leqslant C A P_{j}^{\mathrm{T}}, \quad \forall t \in \mathcal{T}, j \in \mathcal{J} \quad\left(\alpha_{t, j}^{-}, \alpha_{t, j}^{+}\right) \\
& 0 \leqslant g_{t}^{\mathrm{w}} \leqslant L W_{t} \cdot C A P^{\mathrm{W}}, \quad \forall t \in \mathcal{T} \quad\left(\beta_{t}^{-}, \beta_{t}^{+}\right) \\
& 0 \leqslant p v_{t}^{\mathrm{ra}} \leqslant L S_{t} \cdot C A P_{P V}^{\mathrm{ra}}, \quad \forall t \in \mathcal{T} \quad\left(\gamma_{t}^{-}, \gamma_{t}^{+}\right) \\
& e_{t}^{\mathrm{ra}}=e_{t-1}^{\mathrm{ra}}+c h_{t}^{\mathrm{ra}} \cdot E C-d c_{t}^{\mathrm{ra}} / E D, \quad t \in \mathcal{T} \backslash\{1\} \quad\left(\delta_{t}\right) \\
& e_{1}^{\mathrm{ra}}=E_{0}^{\mathrm{ra}}+c h_{1}^{\mathrm{ra}} \cdot E C-d c_{1}^{\mathrm{ra}} / E D, \quad\left(\delta_{1}\right) \\
& e_{T}^{\mathrm{ra}}=E_{0}^{\mathrm{ra}}, \quad(\epsilon) \\
& 0 \leqslant e_{t}^{\mathrm{ra}} \leqslant \overline{E^{\mathrm{ra}}}, \quad \forall t \in \mathcal{T} \quad\left(\zeta_{t}^{-}, \zeta_{t}^{+}\right) \\
& 0 \leqslant c h_{t}^{\mathrm{ra}} \leqslant C R \cdot \overline{E^{\mathrm{ra}}}, \quad \forall t \in \mathcal{T} \quad\left(\eta_{t}^{-}, \eta_{t}^{+}\right) \\
& 0 \leqslant d c_{t}^{\mathrm{ra}} \leqslant C R \cdot \overline{E^{\mathrm{ra}}}, \quad \forall t \in \mathcal{T} \quad\left(\theta_{t}^{-}, \theta_{t}^{+}\right) \\
& 0 \leqslant w_{t}^{\mathrm{ag}} \leqslant \overline{W_{t}^{\mathrm{ag}}}, \quad \forall t \in \mathcal{T} \quad\left(\iota_{t}^{-}, \iota_{t}^{+}\right) \\
& 0 \leqslant i_{t}^{\mathrm{ag}} \leqslant \overline{I_{t}^{\mathrm{ag}}}, \quad \forall t \in \mathcal{T} \quad\left(\kappa_{t}^{-}, \kappa_{t}^{+}\right)
\end{aligned}
$$

Equation (11) represents the power balance at wholesale level. The total offtake, equal to the passive inelastic demand $D_{t}^{\mathrm{ra}}+$ $D_{t}^{\mathrm{rp}}+D_{t}^{\mathrm{i}}$, the power charged to the aggregated ESS of the nonstrategic active consumers of RA $c h_{t}^{\text {ra }}$ and the withdrawal of the aggregator $w_{t}^{\mathrm{ag}}$, should be balanced by the total injection, equal to the generation of thermal power plants $g_{t j}^{\mathrm{t}}$ and wind turbines $g_{t}^{\mathrm{w}}$, the PV production $p v_{t}^{\mathrm{ra}}$ and discharged power $d c_{t}^{\mathrm{ra}}$ of RA and the injection of the aggregator $i_{t}^{\mathrm{ag}}$. The dual variable $\lambda_{t}$ of this power balance constraint is the market clearing price. Constraint (12) limits the power output of each generator to its capacity while Constraint (13) limits the power output of the curtailable wind generation to its capacity multiplied by the load factor $\left(L W_{t}\right)$. Constraint (14) limits the PV production of RA. Constraints (15)-(20) define the operation limits of the aggregated ESS of RA. They correspond to Equations (3)(8) in the UL problem. Last, Eq. (21)-(22) limit the cleared withdrawal/injection quantity associated with the aggregator to its bid $\overline{W_{t}^{\mathrm{ag}}} / \overline{I_{t}^{\mathrm{ag}}}$ (decision variables of the UL problem).

3) Reformulation as MILP: The bilevel problem (1)-(22) can be reformulated as a MILP problem by replacing the LL optimization problem with the associated KKT conditions (stationarity conditions (23)-(31) and complementary slackness conditions (32)-(47)). After linearization (see below), the 
resulting MILP problem (Min. (48) s.t. constraints (2)-(9), (11)-(47)) can be solved using a commercial MILP solver such as Gurobi.

$$
\begin{aligned}
& V C_{j}-\lambda_{t}-\alpha_{t, j}^{-}+\alpha_{t, j}^{+}=0, \quad \forall t \in \mathcal{T} \\
& -\lambda_{t}-\beta_{t}^{-}+\beta_{t}^{+}=0, \quad \forall t \in \mathcal{T} \\
& -\lambda_{t}-\gamma_{t}^{-}+\gamma_{t}^{+}=0, \quad \forall t \in \mathcal{T} \\
& -\delta_{t}+\delta_{t+1}-\zeta_{t}^{-}+\zeta_{t}^{+}=0, \quad t \in \mathcal{T} \backslash\{1\} \\
& -\delta_{T}-\epsilon-\zeta_{T}^{-}+\zeta_{T}^{+}=0, \\
& \lambda_{t}+\delta_{t} \cdot E C-\eta_{t}^{-}+\eta_{t}^{+}=0, \quad \forall t \in \mathcal{T} \\
& -\lambda_{t}-\delta_{t} / E D-\theta_{t}^{-}+\theta_{t}^{+}=0, \quad \forall t \in \mathcal{T} \\
& -P^{c a p}+\lambda_{t}-\iota_{t}^{-}+\iota_{t}^{+}=0, \quad \forall t \in \mathcal{T} \\
& -\lambda_{t}-\kappa_{t}^{-}+\kappa_{t}^{+}=0, \quad \forall t \in \mathcal{T} \\
& 0 \leq \alpha_{t, j}^{-} \perp g_{t, j}^{t} \geq 0, \quad \forall t \in \mathcal{T}, \forall j \in \mathcal{J} \\
& 0 \leq \alpha_{t, j}^{+} \perp C A P_{j}^{T}-g_{t, j}^{t} \geq 0, \quad \forall t \in \mathcal{T}, \forall j \in \mathcal{J} \\
& 0 \leq \beta_{t}^{-} \perp g_{t}^{w} \geq 0, \quad \forall t \in \mathcal{T} \\
& 0 \leq \beta_{t}^{+} \perp L W_{t} \cdot C A P^{W}-g_{t}^{w} \geq 0, \quad \forall t \in \mathcal{T} \\
& 0 \leq \gamma_{t}^{-} \perp p v_{t}^{\mathrm{ra}} \geq 0, \quad \forall t \in \mathcal{T} \\
& 0 \leq \gamma_{t}^{+} \perp L S_{t} \cdot C A P_{P V}^{\mathrm{ra}}-p v_{t}^{\mathrm{ra}} \geq 0, \quad \forall t \in \mathcal{T} \\
& 0 \leq \zeta_{t}^{-} \perp e_{t}^{\mathrm{ra}} \geq 0, \quad \forall t \in \mathcal{T} \\
& 0 \leq \zeta_{t}^{+} \perp \overline{E^{\mathrm{ra}}}-e_{t}^{\mathrm{ra}} \geq 0, \quad \forall t \in \mathcal{T} \\
& 0 \leq \eta_{t}^{-} \perp c h_{t}^{\text {ra }} \geq 0, \quad \forall t \in \mathcal{T} \\
& 0 \leq \eta_{t}^{+} \perp C R \cdot \overline{E^{\mathrm{ra}}}-c h_{t}^{\mathrm{ra}} \geq 0, \quad \forall t \in \mathcal{T} \\
& 0 \leq \theta_{t}^{-} \perp d c_{t}^{\mathrm{ra}} \geq 0, \quad \forall t \in \mathcal{T} \\
& 0 \leq \theta_{t}^{+} \perp C R \cdot \overline{E^{\mathrm{ra}}}-d c_{t}^{\mathrm{ra}} \geq 0, \quad \forall t \in \mathcal{T} \\
& 0 \leq \iota_{t, j}^{-} \perp w_{t, j}^{\mathrm{ag}} \geq 0, \quad \forall t \in \mathcal{T} \\
& 0 \leq \iota_{t, j}^{+} \perp \overline{W_{t}^{\mathrm{ag}}}-w_{t}^{\mathrm{ag}} \geq 0, \quad \forall t \in \mathcal{T} \\
& 0 \leq \kappa_{t, j}^{-} \perp i_{t, j}^{\mathrm{ag}} \geq 0, \quad \forall t \in \mathcal{T} \\
& 0 \leq \kappa_{t, j}^{+} \perp \overline{I_{t}^{\mathrm{ag}}}-i_{t}^{\mathrm{ag}} \geq 0, \quad \forall t \in \mathcal{T}
\end{aligned}
$$

The complementary slackness conditions (32)-(47) are linearized following the Big-M Method of Fortuny-Amat and McCarl [9]. The non-linear term $\lambda_{t} \cdot\left(w_{t}^{\mathrm{ag}}-i_{t}^{\mathrm{ag}}\right)$ in the objective (1) is linearized using Eq. (11), (23)-(29) and (32)-(43):

$$
\begin{aligned}
& \text { Minimize } \sum_{t \in \mathcal{T}}\left(-\lambda_{t} \cdot\left(D_{t}^{\mathrm{rp}}+D_{t}^{\mathrm{ra}}+D_{t}^{\mathrm{i}}\right)\right. \\
& +\sum_{j \in \mathcal{J}}\left(V C_{j} \cdot g_{t, j}^{\mathrm{t}}+\alpha_{t, j}^{+} \cdot C A P_{j}^{\mathrm{T}}\right)+\beta_{t}^{+} \cdot L W_{t} \cdot C A P^{\mathrm{W}} \\
& \left.+\overline{E^{\mathrm{ra}}} \cdot\left(\zeta_{t}^{+}+C R\left(\eta_{t}^{+}+\theta_{t}^{+}\right)\right)+\gamma_{t}^{+} \cdot L S_{t} \cdot C A P_{P V}^{\mathrm{ra}}\right) \\
& -E_{0}^{\mathrm{ag}} \cdot\left(\delta_{1}+\epsilon\right)+\text { distcosts }^{\mathrm{ag}}
\end{aligned}
$$

\section{CASE STUDY}

The current Belgian power system is taken as a starting point, but the $6 \mathrm{GW}$ of nuclear generation is replaced by gasfired power plants, the installed capacity of wind and solar is doubled and energy storage is added. This results in $12.7 \mathrm{GW}$

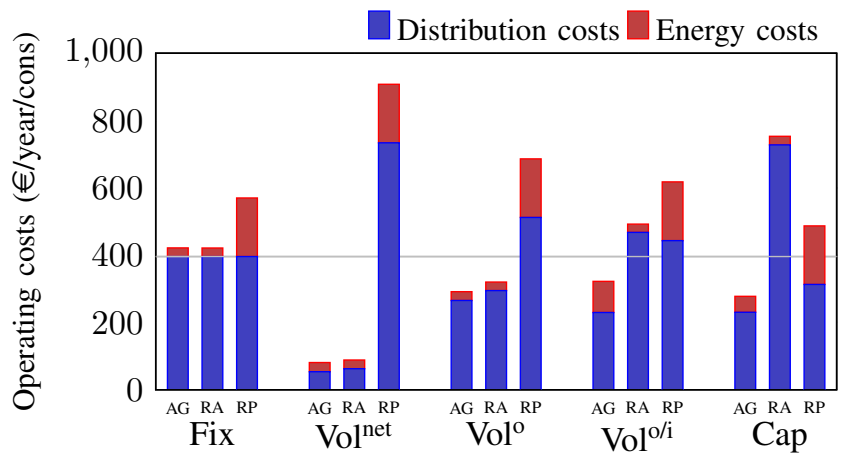

Fig. 1: The average yearly operating costs per consumer for the aggregator (AG), the retailer with active consumers (RA) and the retailer with passive consumers (RP) under five different distribution tariff designs.

of thermal generation capacity (mainly combined-cycle and open-cycle gas turbines), $6 \mathrm{GW}$ of wind generation capacity, $8 \mathrm{GW}$ of solar PV capacity and $3 \mathrm{GWh}$ of storage capacity. The ESS have a C-Rate of 0.33, i.e., they can be fully charged or discharged in three hours. The charging and discharging efficiencies are $95 \%$. We assume all solar and storage capacity is uniformly distributed among all active residential consumers while the wind turbines and thermal generators are owned by generation companies.

The three suppliers (RP, RA and AG) serve a total of 4.8 million residential consumers. Of those consumers, $50 \%$ are assumed passive and thus supplied by RP while $25 \%$ are supplied by RA and $25 \%$ are supplied by AG. The load curves of RP, RA and AG are obtained using the 2017 synthetic load profiles (SLPs) of residential consumers with a yearly demand of $3500 \mathrm{kWh}$, which is the average electricity demand of a household in Belgium [10]. The non-residential demand is calculated by subtracting these SLPs from the total load of the Belgian power system in 2017, provided by the Belgian TSO Elia [11]. The annual residential demand equals 16.8 TWh, compared to a total load of 77.3 TWh.

To be able to compare the different tariff designs on equal footing, each tariff must be set in such a way that the DSO always recovers $1900 \mathrm{M} € /$ year or $400 € /$ year/consumer on average from residential consumers [1]. We do not take into account cost recovery from the industrial consumers. It is not straightforward to predict the recovered costs due to the strategic behavior of the aggregator who tries to avoid them, but they can be calculated after running the model. By iteratively updating the exogenous tariff, a tariff can be found which ensures that the DSO recovers the required amount for each tariff design. All simulations are performed on a set of 12 representative days of the year 2017, selected using the algorithm developed by Poncelet et al. [12].

First, we discuss the impact of the five distribution tariff designs on the annual operating costs of the retailers RP and RA and the aggregator AG (Section III-A). Second, we present a sensitivity analysis w.r.t. the share of active consumers with AG and the total amount of ESS in the system (Section III-B). 

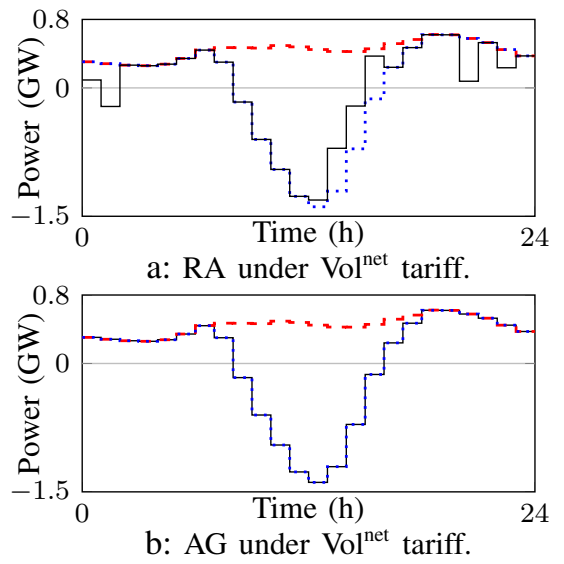

- - - $D_{t} \cdots \cdots \cdot D_{t}-p v_{t}-D_{t}-p v_{t}+c h_{t}-d c_{t}$
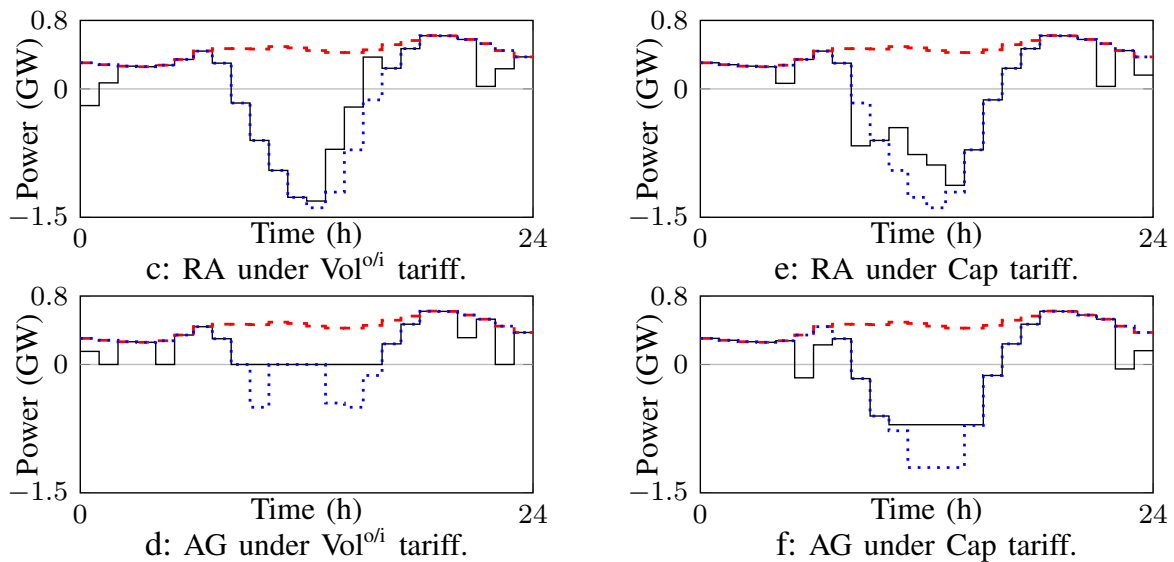

Fig. 2: The residual demand curve (black line) is obtained subtracting the PV production from the original demand curve (dashed red line) and adding/subtracting the power charged to/discharged from the ESS. The residual demand is equal to the net offtake (if it is positive) or injection (if it is negative). As an intermediate result, the original demand corrected for the PV production is shown (dotted blue line).

\section{A. Results \& discussion}

Figure 1 shows the yearly operating costs of RP, RA and AG divided by the number of customers each entity serves. RA has lower operating costs under most tariff designs compared to RP. These lower operating costs, however, will typically not enable RA to increase its profit margin compared to RP as the decrease in costs is due to the investment of consumers in PV and ESS, which lowers their residual demand. Furthermore, the operating costs of AG are usually lower than those of RA. In this case, the decrease is not due to the investments of the consumers (the active consumers of AG have done exactly the same investment as the active consumers of RA), but due to the strategic behavior of the aggregator. This implies that the aggregator may be able to increase its profit margin compared to the retailers. Figure 1 illustrates that the distribution tariff design has a significant impact on the results, which are analyzed in detail per tariff design below.

1) Fixed tariff: Under a fixed distribution tariff, only energy costs can be decreased. Figure 1 shows that investments in PV and ESS greatly reduce these energy costs for active consumers (RA and AG) compared to passive consumers (RP). This is mainly due to a decrease of the residual demand of these consumers. Note that PV generation and arbitrage with storage can also decrease wholesale market prices, but this affects all consumers equally, i.e., all consumers face the same electricity price profile. The strategic aggregator cannot reduce its energy costs compared to RA as the non-strategic consumers of RA operate their PV in ESS in a way that is optimal for the system. This strategy always leads to the lowest energy costs, but these "naive" consumers do not take into account their distribution costs which can be detrimental under other tariff structures.

2) Volumetric tariff with net-metering: Active consumers can greatly reduce their costs by transferring a significant share of the distribution costs to passive consumers while also decreasing their energy costs. In this case, the strategic aggregator can reduce his operating costs compared to RA by further lowering the distribution costs (from $62.4 € /$ year/cons to $53.5 € /$ year/cons) and slightly increasing the energy costs (from $25.9 € /$ year/cons to $27.3 € /$ year/cons). Figures $2 \mathrm{a}$ and $2 \mathrm{~b}$, displaying the residual demand curves during one day of RA and AG respectively, show how the aggregator manages this. Contrary to the active consumers of RA, the aggregator does not use its ESS in order to avoid efficiency losses. Due to these losses, the total amount of charged electricity is always higher than the total amount of discharged electricity. As a result, charging and discharging the ESS always increases the net offtake, which increases the distribution costs. The benefit of arbitraging with the ESS, i.e. lower energy costs, does not outweigh the decrease in distribution costs that can be attained by barely using the ESS. The gains of AG compared to RA are however small, as the main decrease in net offtake, hence energy and distribution costs, is driven by PV generation.

3) Volumetric tariff on hourly offtake: Active consumers can again transfer distribution costs to passive consumers, but to a lesser extent compared to the volumetric tariff with netmetering. In this case, injection during hours when PV generation is high, does not aid in decreasing the distribution costs as the offtake is not netted over the whole year. The aggregator strategically operates its ESS to lower the distribution costs with $30.6 € /$ year/cons compared to RA, while only slightly increasing the energy costs $(0.8 € /$ year/cons $)$. In contrast to the retailer, the aggregator never withdraws electricity to charge its ESS, since this would increase its distribution costs.

4) Volumetric tariff on hourly offtake and injection: The energy costs of RA are significantly lower than the energy costs of RP (Fig. 1). However, the distribution costs of RA are higher than those of RP because active consumers are penalized for injection. As mentioned earlier, the non-strategic consumers of RA do not take into account their distribution costs. The aggregator, on the other hand, can obtain large cost savings of $170.1 € /$ year/cons compared to RA by focusing on jointly minimizing the energy and distribution costs. To 

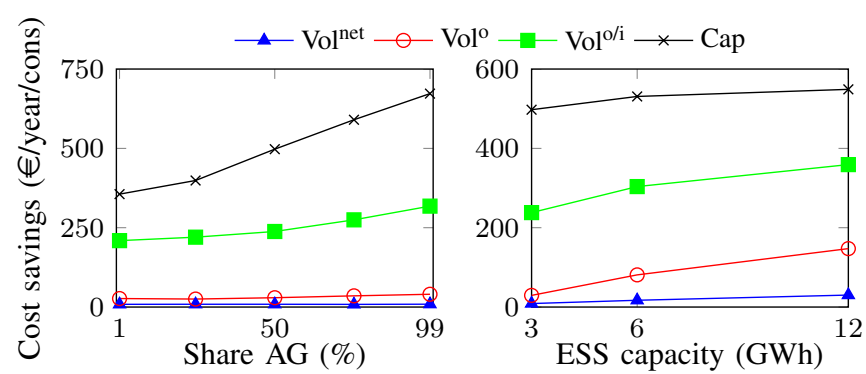

a: Distribution cost savings.

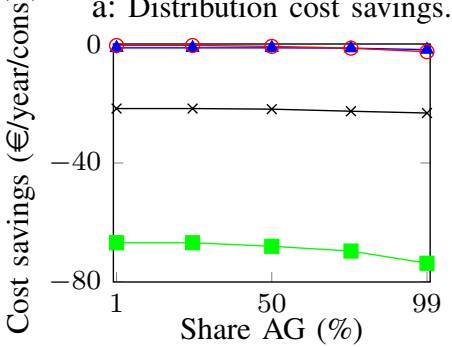

c: Distribution cost savings.

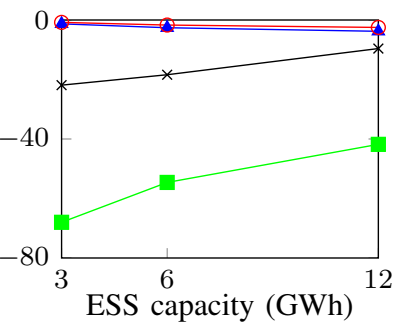

b: Energy cost savings.

in the system. When AG owns more ESS, it has more options to strategically lower its operating costs.

These sensitivities indicate that, qualitatively, the relative performance of the aggregator, in terms of operating cost savings compared to the retailer, between different tariff designs is always similar: the qualitative results illustrated by Fig. 1 can be generalized for different shares of active consumers with the aggregator and different total ESS capacities.

\section{CONCLUSION}

Aggregators may bring the flexibility of residential consumers' distributed energy storage systems to the wholesale market. When optimizing the operation of these distributed resources, an aggregator may take into account the distribution costs as this is often the largest component of the electricity bill of a residential consumer. In this paper, we represent the interaction between a strategic aggregator of residential consumers with PV and ESS and a wholesale market as a Stackelberg game, formulated as a bilevel optimization problem. The aggregator explicitly takes into account energy and distribution costs for supplying its consumers.

In a case study, inspired by the Belgian power system, we show how the aggregator decreases its operating costs compared to a retailer of active consumers by avoiding distribution costs. The aggregator attains the highest operating cost savings under a capacity tariff, followed by a volumetric tariff on offtake and injection, a volumetric tariff on offtake and, last, a volumetric tariff with net-metering. Under a fixed distribution tariff, the aggregator cannot save costs as its energy costs are never lower than those of the retailer.

\section{REFERENCES}

of RP because RA pays the largest share of distribution costs. Again, the active consumers of RA do not take into account their distribution costs. As their injection peak is higher than the offtake peak of passive consumers, this leads to very high distribution costs for RA, which outweigh the energy savings. Figure 2f, which can be compared to Figure 2e, shows how the aggregator obtains large distribution cost savings by curtailing its PV generation and charging its ESS to reduce its injection peak. This causes the energy costs to increase (compared to RA), but the decrease in distribution costs leads to overall cost savings of $475.6 €$ /year/cons compared to RA.

\section{B. Sensitivity analysis}

An increasing share of active consumers contracted by AG leads to increasing operating cost savings for AG compared to RA for every tariff design except $\mathrm{Vol}^{\text {net }}$ where they remain constant. This follows from increasing distribution cost savings (Fig. 3a) while the energy cost savings (note that they are always negative) decrease at a slower rate (Fig. 3b). When more active consumers become strategic and start avoiding distribution costs, the tariffs will increase to ensure cost recovery of the DSO. This leads to increased distribution costs for RA and AG. The increase for AG is however smaller as it attempts to avoid these costs. Furthermore, Figures $3 \mathrm{c}$ and $3 \mathrm{~d}$ illustrate that the operating cost savings of AG compared to RA also increase as a function of the total storage capacity

[1] VREG, "Marktmonitor 2017," tech. rep., Brussels, Belgium, 2018.

[2] CEER, "Electricity distribution network tariffs ceer guidelines of good practice," tech. rep., Brussels, Belgium, 2017.

[3] J. Jargstorf, Capacity-Based Residential Grid Tariffs in the Context of Photovoltaic Generation and Battery Storage. PhD thesis, KU Leuven, 2016.

[4] T. Schittekatte, I. Momber, and L. Meeus, "Future-proof tariff design: Recovering sunk grid costs in a world where consumers are pushing back," Energy Economics, vol. 70, no. 2, pp. 484-498, 2018.

[5] S. Borenstein, "The economics of fixed cost recovery by utilities," The Electricity Journal, vol. 29, no. 7, pp. 5-12, 2016.

[6] M. G. Vayá and G. Andersson, "Optimal bidding strategy of a plugin electric vehicle aggregator in day-ahead electricity markets under uncertainty," IEEE Trans. Power Syst., vol. 30, no. 5, pp. 2375-2385, 2015.

[7] E. G. Kardakos, C. K. Simoglou, and A. G. Bakirtzis, "Optimal offering strategy of a virtual power plant: A stochastic bi-level approach," IEEE Trans. Smart Grid, vol. 7, no. 2, pp. 794-806, 2016.

[8] K. Bruninx, H. Pandžić, H. Le Cadre, and E. Delarue, "On Controllability of Demand Response Resources \& Aggregators' Bidding Strategies," Accepted for PSCC 2018, Dublin, Ireland, June 11-15, 2018.

[9] J. Fortuny-Amat and B. McCarl, "A representation and economic interpretation of a two-level programming problem," Journal of the operational Research Society, vol. 32, no. 9, pp. 783-792, 1981.

[10] Synergrid, "Synthetic load profiles." http://www.synergrid.be/index.cfm? PageID=16896\&language_code=NED\#. Accessed 27-03-2018.

[11] Elia, "Data download." http://www.elia.be/nl/grid-data/data-download. Accessed 27-03-2018.

[12] K. Poncelet, H. Höschle, E. Delarue, A. Virag, and W. Dhaeseleer, "Selecting representative days for capturing the implications of integrating intermittent renewables in generation expansion planning problems," IEEE Trans. Power Syst., vol. 32, no. 3, pp. 1936-1948, 2017. 